\title{
Improvement of Power System Small-Signal Stability by Artificial Neural Network Based on Feedback Error Learning
}

\author{
Sedat NAZLIBILEK*, Issa ALI, Alyaseh ASKIR
}

\begin{abstract}
Electrical power systems usually suffer from instabilities because of some disturbances occurring due to environmental conditions, system failures, and loading conditions. The most frequently encountered problem is the loss of synchronization between the rotor angle and the stator magnetic angle for synchronous generators. The contribution of this study is that a nonlinear adaptive control approach called feedback error learning (FEL) is utilized to improve the small-signal stabilities of an electric power system. The power system under study is composed of a synchronous machine connected to infinite bus. Many advantages of FEL control approach makes it capable to robustly adapting with all possible operating conditions rather than using optimization algorithms for tuning the conventional power system stabilizer (CPSS) that is still unsatisfactory especially at some critical operating points. The performances of two controllers, namely the proposed FEL scheme and the conventional controller CPSS, are tested by Matlab simulations. It is found that the FEL controller can be effectively used as an alternative stabilizer for improving small-signal stabilities of the power system.
\end{abstract}

Keywords: artificial neural network; conventional PSS; conventional power system stabilizer; feedback error learning; SMIB power system

\section{INTRODUCTION}

Electrical power system supplying electricity to facilities, commercial and residential users, is normally a huge and complex system. It may be considered as a system of systems. A power system is composed of drivers or prime movers, generators, transmission systems, subtransmission systems, and loads. The system has to be reliable and sustainable and to supply electrical energy or power to the users continuously. The system must satisfy a constant frequency and a constant voltage in an uninterruptable fashion. However, electrical power systems usually suffer from instabilities because of some disturbances occurring because of environmental conditions, system failures, and loading conditions. The most frequently encountered problem is the loss of synchronization between the rotor angle and the stator magnetic angle for synchronous generators [1]. We describe this type of problem as small signal instability. The definitions and also the classifications of the terms on power system stability are given by IEEE/CIGRE JTF [2]. The Joint Task Force has published all the terms and definitions on power systems stability such as voltage stability, frequency stability, small-signal stability, transient stability, oscillatory stability, and Lyapunov stability. We use the definition of power system stability given by this Joint Task Force. The contribution of this study is that a very effective control method called feedback error learning (FEL) is used to improve the smallsignal stabilities of an electric power system that is composed of a synchronous generator and an infinite bus connected to it, as it is well known that power system stabilizers (PSS) are used to damp the oscillations for the small signal stability. However, local signal based controls are the first measure for power system stability, they are the important stage of effectively damping inter area modes $[3,4]$. Because of the lack of observability of the system modal characteristics, the use of power system stabilizers (PSSs) has been the first measure to enhance small signal stability by damping oscillations. Stability and control problems of power generating systems have been tackled since the beginning of twenties [5-7] in which R. H. Park also published his invention on some means for maintaining power systems stability under fault conditions [8]. Several scientific, practical engineering books on power system stability have been published as well since 50 's $[9,10]$. Studies on the important problem of dynamic stability of power systems have been going on for about hundred years. Stability of single synchronous generators connected to infinite bus is widely studied in [11]. Some other researches as in [12] used the power systems sensitivity model such that the infinite bus representation was not needed. The model was first derived for a single machine infinite bus (SMIB) and the performance was then compared with the classical Heffron-Phillips model. Small-signal stability margins for electromechanical modes were studied in [13-15]. In these studies, electromechanical modes were estimated by phasor measurement units (PMUs) under ambient conditions. In [16], a new algorithm was proposed for the estimation of electromechanical modes under forced oscillations. However, finding the location of force oscillations is not an easy task. There are some studies for locating them, described in the literature [17]. Another important area of stability is related to robust stability analysis of power systems [18]. In recent years, genetic algorithms, intelligent methods and learning algorithms have been applied to solve stability problems that cannot be achieved with the use of classical control methods [19-22]. A power system on-line instability monitoring system was proposed using convolutional neural network (CNN) in [23] which is the first study applying $\mathrm{CNN}$ in such an application. Prediction of instability for rotor angle was studied by a couple of authors who used post-disturbance voltage trajectories [24]. An adaptive ANN was applied to predict rotor angle stability of generator for dynamic security assessment in [25]. In order to control frequency of loads for stochastic power systems, a deep reinforced learning method is proposed in [26]. Although these studies were done to obtain robust systems and better operating performance, they still have some difficulties for achieving stability in some operating conditions. An effective controller called feedback error learning scheme was first proposed in [27]. Although it was first applied to a robotic 
problem, the approach was used for a couple of different applications as well [28]. Discrete-time FEL theory was presented in [29] and its application as a nonlinear adaptive controller in [30]. The contribution of our study is in the use of the artificial intelligent technique within the framework of feedback error learning control scheme in order to improve the small-signal stability of the power system composed of a synchronous machine connected to an infinite Bus. We use the Self-Adaptive Discrete Time MIMO Linear Neural Network (ADALINE) tool for this study $[31,32]$. The results obtained by testing the FEL controller are compared with the conventional power system stabilizer. It was seen that the proposed control approach has a better performance than the classical stabilizer. The system model is explained in Section 2. The classical controller is given in Section 3. The proposed control scheme is described in Section 4. Finally, the simulation results are presented in Section 5.

\section{SYSTEM MODEL}

The power system block diagram is shown in Fig. 1. It consists of a synchronous generator, an external exciter, a driver that is a combination of a turbine and governor, a power system stabilizer (PSS), an automatic voltage regulator (AVR) and an infinite bus to which the synchronous machine is connected. It is a single machine and infinite bus (SMIB) system.

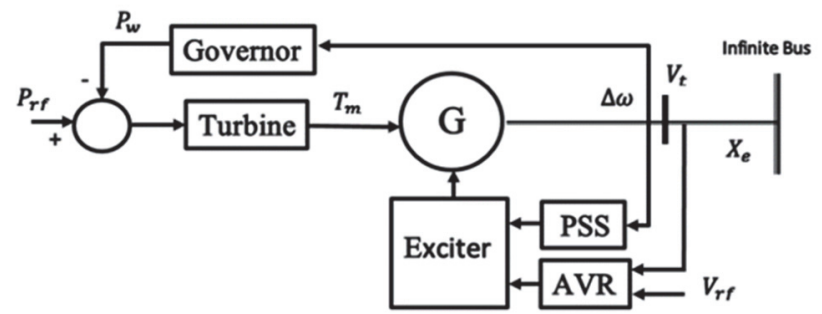

Figure 1 Schematic block diagram of SMIB with control and excitation components

The automatic voltage regulator (AVR) and the power system stabilizer (PSS) are normally used for small-signal stabilization of the generator. The AVR and the PSS are responsible for two actions. One of them is to damp the low frequency oscillations and the other one is to keep the voltage in a specific desired level. The AVR amplifies the input signals and stabilizes the exciter. The PSS gives some extra signals such as frequency and rotor speed deviations, $\Delta \mathrm{f}$ and $\Delta \omega$ respectively and power for acceleration to the AVR. It is responsible to damp oscillations at the rotor of the generator [1]. The dynamical system representation of the synchronous generator is given as a set of linear differential Eq. (1) to Eq. (4) [1] as follows:

$$
\begin{aligned}
& \dot{\delta}=\omega-\omega_{0} \\
& \dot{\omega}=\frac{1}{2 H}\left(-D \omega+T_{m}-T_{e}\right) \\
& \dot{E}_{q}=\frac{1}{T_{d o}}\left(-E_{q}+\left(X_{d}-\dot{X}_{d}\right) i_{d}+E_{f d}\right)
\end{aligned}
$$

$$
\dot{E}_{d}=\frac{1}{T_{q o}}\left(-E_{d}+\left(X_{q}-\dot{X}_{q}\right) i_{q}\right)
$$

where $\delta$ is the angular position of the rotor in electrical radians, $\omega$ is the angular velocity of the rotor in electrical $\mathrm{rad} / \mathrm{s}, \omega_{0}$ is the rated angular velocity, $T_{m}$, is the input mechanical torque, $T_{e}$, is the induced electromagnetic torque, $H$ is the inertia constant, $D$ is the damping coefficient. The current $i_{d}$ and $i_{q}$ are the components of armature currents in direct axis and quadrature axis respectively. $E_{f d}$ is the field voltage. $E_{d}$ and $E_{q}$ are the components of the terminal voltage in direct and quadrature axes respectively. $T_{d o}$ and $T_{q o}$ are the opencircuit transient time constants in $d$-axis and $q$-axis respectively. $X_{d}$ and $X_{q}$ are the transient reactances in $d$ and $q$-axis respectively. To analyse the system described by the authors in Eq. (1) to Eq. (4), the linearized Heffron Phillips model in the following state space representation is used [1].

$$
\begin{aligned}
& \Delta \dot{\delta}=\omega_{0} \Delta \omega \\
& \Delta \dot{\omega}=\frac{1}{2 H}\left(-K \Delta \delta-D \Delta \omega-K_{2} \Delta E_{q}+\Delta T_{m}\right) \\
& \Delta \dot{E}_{q}=\frac{1}{T_{d o}}\left(-K_{4} \Delta \delta-\frac{1}{K_{3}} \Delta E_{q}+E_{f d}\right) \\
& \Delta \dot{E}_{f d}=\frac{1}{T_{A}}\left(-K_{4} K_{5} \Delta \delta-K_{A} K_{6} \Delta E_{q}-E_{f d}+K_{A} u\right)
\end{aligned}
$$

where $\Delta$ denotes a small deviation in states, and the constants $\left(K_{1} \div K_{6}\right)$ are known as Heffron Phillips constants that describe the internal influence factors within the system as follows [25]. $K_{1}$ and $K_{2}$ represent the influences of torque angle and internal voltage respectively on electric torque. $K_{3}$ represents the influence of field winding. $K_{4}$ and $K_{5}$ represent the influences of the torque angle on the field voltage and the terminal voltage respectively. $K_{6}$ represents the influence of the internal voltage on the terminal voltage. $K$ constants are basically functions of the real power $P$ and the reactive power $Q$ produced by the synchronous machine and they are also affected by the system impedance and terminal voltage as well. The expressions used to determine the values of $K$ constants can be found in [26].

\section{CONVENTIONAL POWER SYSTEM STABILIZER}

Because of the simplicity of its design and implementation, the so called classical power system stabilizers have been widely used for many years in order to stabilize power systems. The stabilizer is composed of three parts. The first part is an amplifier with a gain $K_{p s s}$. The second part is a washout filter with a time constant, $T_{w}$ which serves as a high pass filter in order to eliminate the undesired effects at steady state conditions. The third part is a phase compensator with time constants $T_{1}$ and $T_{2}$. It is used as a phase lag compensator between the exciter input and the electrical torque of the synchronous generator [1]. 
The input signal of the CPSS is the speed deviation of the machine shaft while the output signal is a supplementary stabilizing signal.

\section{FEEDBACK ERROR LEARNING CONTROL SCHEME 4.1 Learning Mechanisms in System Control}

Artificial intelligence is a very popular subject nowadays. Among several approaches, the well-known and mostly applied technique is the neural network structures. There are a lot of neural network structures studied and used in the literature. Although the neural networks have been used for more than forty years, some deep learning networks have been introduced recently. The feedback error learning scheme is one of the approaches used for control applications. It was introduced by Kawato et al [27] as architecture for robotic application for voluntary motion. It was a computation model to generate motor command similar to animal brain. They propose a learning model implementing inverse model of muscles and skeletal system. The FEL control scheme is a combination of two controllers such that one of them is a feed forward neural network controller and the other is a classical feedback controller with fixed gain. The neural network is trained online by using the output of the classical controller and the plant itself. After a certain learning period, the neural network takes the role as an adaptive controller instead of the conventional one resulting in a system that is more robust against structural uncertainties and external disturbances. Several neural network and feedback error learning schemes were applied to control problems [28-30]. Block diagram of the feedback error learning scheme is depicted in Fig. 2.

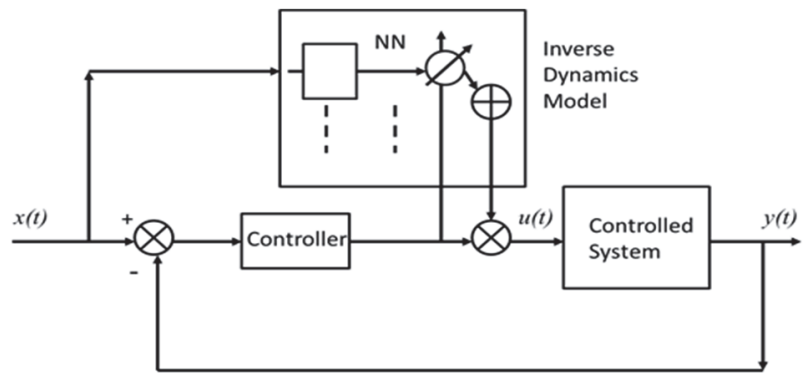

Figure 2 Feedback Error Learning control scheme

\subsection{Matlab Adaptive Neural Network Library}

The Neural Network Tool Box supports supervised learning with feed-forward and dynamic networks under Matlab environment [36]. However, such product is still not satisfactorily integrated with Simulink hence the neural network subsystems that are developed in Matlab environment are sometimes slow and not effective with real time applications. For the purpose of overcoming these difficulties, "Adaptive Neural Network Library" was developed at the West Virginia University [32]. The library includes different kinds of adaptive neural networks. A "Self-Adaptive Discrete Time MIMO Linear Neural Network" (ADALINE) tool has been used for this study. ADALINE network block has three input channels namely the input vector, $x n$, the error, $e$, and the logical enable signal $L E$ enabling the learning as " 1 " or disabling it as " 0 ". The output channels of the network are both the main output $y$ and the state vector $\boldsymbol{x}$ and its entries are the internal states of the network comprising the weights and the parameters that are adjusted during the learning process.

\subsection{Application of FEL Controller on SMIB}

The MATLAB/SIMULINK model (Fig. 3) was realized to study the deviation of rotor speed of the power control system described in Section 2.

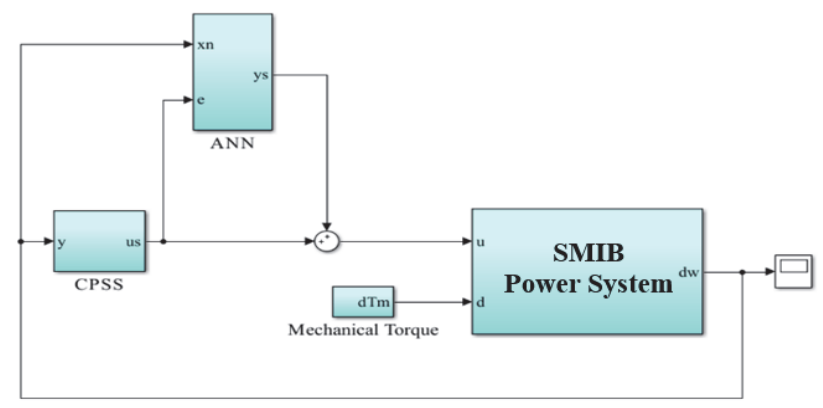

Figure 3 Block diagram of system simulation model

As shown in Fig. 3, two controllers are joined together to form FEL control scheme. The neural controller is a network learning on-line the inverse dynamics of the system. The other controller is a fixed-gain CPSS.

The nominal parameters of the SMIB power system along with the data of applied conventional power system stabilizer have been taken from the system considered in $[33,34]$.

In Fig. 3, the ADALINE Neural Network inputs are the deviation of rotor speed and its time derivative while the network output is a supplementary signal added to the control signal provided by the CPSS. The artificial neural network library provides an interface to set the parameters of ADALINE network. These parameters are set as follows: number of input neurons is 2 , number of output neurons is 1 , number of hidden layers is 1 , number of neurons in hidden layer is 3, Learning rate is 0,7 and Stabilizing Factor is $10^{-9}$, Weight Limiter is $10^{4}$ and finally the sampling time is $10^{-5} \mathrm{~s}$.

\section{SIMULATION RESULTS}

In this section, the simulation results of the power system under study are presented. All simulations are done in Matlab/Simulink environment. In order to demonstrate the effectiveness of the proposed ANN based on FEL control scheme, the system dynamic performance with the proposed controller has been compared to the system dynamic performance with the conventional PSS that was optimally designed in [33].

Table 1 The Simulation Case Studies

\begin{tabular}{|c|c|}
\multicolumn{2}{c}{ Table 1 The Simulation Case Studies } \\
\hline Case & Load $(P, Q) \mathrm{p} . \mathrm{u}$ \\
\hline 1 & Light $(0,5 ; 0,0), t<4 \mathrm{~s}$ \\
Normal $(0,9 ; 0,3), t \geq 4 \mathrm{~s}$
\end{tabular}

The comparison is done by means of considering three cases of system loading listed in Tab. 1. Furthermore, the 
change of mechanical torque $\Delta \mathrm{Tm}$ provided by the prime mover is considered as an external disturbance and applied during simulation process for all considered cases as follows:

$\Delta T_{m}=\left\{\begin{array}{cc}0 \%, & t<0,5 \mathrm{~s} \\ 100 \%, & t \geq 0,5 \mathrm{~s}\end{array}\right.$

It is essential to note that, for all considered cases, the parameter values of ADALINE neural network remains fixed as they have been chosen in section 5 as well as the parameter values of the optimally tuned CPSS controller fixed as designed in [33].

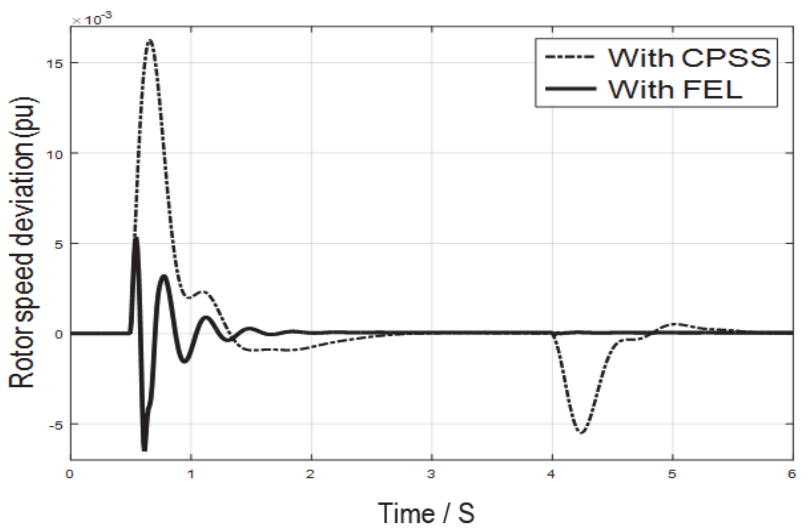

Figure 4 Rotor speed deviation for case 1

The speed deviation of the rotor, in Case 1, is depicted in Fig. 5. In this case, a $100 \%$ step change of $\Delta T_{m}$ is applied to the system at $t=0,5 \mathrm{~s}$; while the operating point is changed from light load to normal load at $t=4 \mathrm{~s}$. It can be seen that the FEL approach is better than the CPSS. The maximum overshoot at $\mathrm{t}=0,5 \mathrm{~s}$ is much lower when the FEL algorithm is applied as the controller. Although there are some oscillations before settling the steady-state when the FEL controller is in the loop, the amplitudes of the oscillations are relatively small and the settling time is also shorter than the CPSS. When the system is switched from light load to normal load, the change in response is so small that it cannot be realized from the graphics at $t=4 \mathrm{~s}$. However, the response of CPSS has a considerable undershoot at that time.

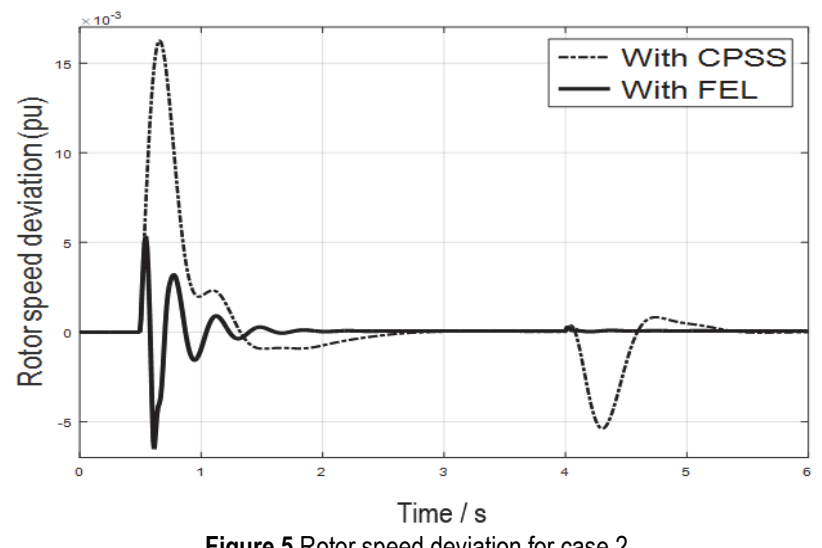

Figure 5 Rotor speed deviation for case 2
Fig. 5 shows the speed deviation of the rotor in Case 2 where a $100 \%$ step change of $\Delta T_{m}$ is applied to the system at $t=0,5 \mathrm{~s}$ while the operating point is changed from light load to heavy load at $t=4 \mathrm{~s}$. Similar results are obtained for Case 2 as well. The step response in transient interval and the load change behavior at $t=4 \mathrm{~s}$ are all better than CPSS when the FEL controller is used.

Finally, Fig. 6 shows the speed deviation of the rotor, in Case 3 where a $100 \%$ step change of $\Delta T_{m}$ is applied to the system at $t=0,5 \mathrm{~s}$, while the operating point is changed from light load to heavy-lead power factor load at $t=4 \mathrm{~s}$. The transient response at $t=0,5 \mathrm{~s}$ is the same as before. However, the considerable load change from a light load to a heavy-lead power factor load makes the system unstable in the case of CPSS controller at $t=4 \mathrm{~s}$. The FEL controller has a very small undershoot at that time and no instability.

The system response settles immediately within half of a second with so little amplitudes of oscillations.

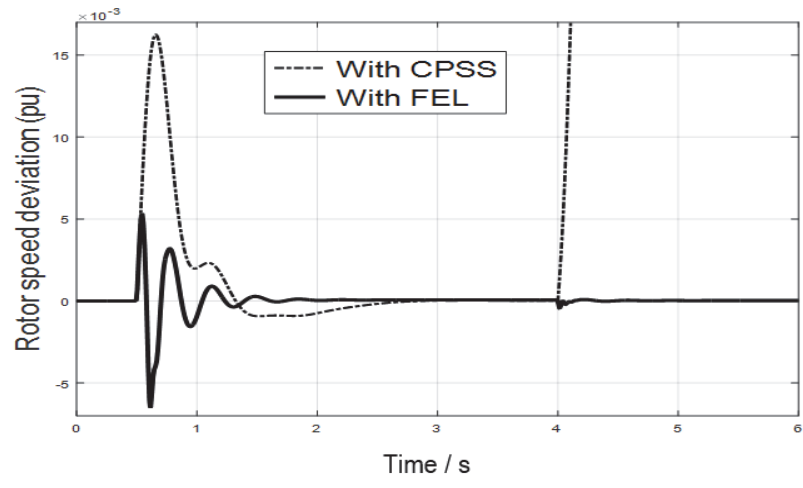

Figure 6 Rotor speed deviation for case 3

\subsection{System Performance Analysis}

The simulation results presented and discussed in the previous section can be numerically summarized through utilizing the three commonly used performance indices which are Integral Squared Error (ISE) defined as $I S E=\int|\mathrm{e}|^{2} \mathrm{~d} t$, Integral Absolute Error $(I A E)$ defined as $I A E=\int|\mathrm{e}| \mathrm{d} t$, and Integral Time-weighted Absolute Error $(I T A E)$ defined as ITAE $=\int|\mathrm{e}| t \mathrm{~d} t$. The numerical values of these indices are calculated for each case study and listed in Tab. 2. $4,41 \times 10^{-6}$

Table 2 The Performance Indexes

\begin{tabular}{|c|c|c|c|c|c|c|}
\hline \multirow{2}{*}{\begin{tabular}{c} 
总 \\
\cline { 2 - 7 }
\end{tabular}} & \multicolumn{2}{|c|}{$I S E$} & \multicolumn{2}{c|}{$I A E$} & \multicolumn{2}{c|}{ ITAE } \\
\cline { 2 - 7 } & ANN & CPSS & ANN & CPSS & ANN & CPSS \\
\hline 1 & $4,41 \times 10^{-6}$ & $5,48 \times 10^{-5}$ & $16,6 \times 10^{-4}$ & 0,007 & 0,019 & 0,137 \\
\hline 2 & $4,41 \times 10^{-6}$ & 0,110 & $16,2 \times 10^{-3}$ & 0,310 & 0,017 & 1,730 \\
\hline 3 & $4,41 \times 10^{-6}$ & inf & $10,3 \times 10^{-3}$ & inf & 0,018 & inf \\
\hline
\end{tabular}

The numerical results depicted in Tab. 2 show the robustness of the proposed control scheme where the conventional stabilizer had poor performance with changing of operating conditions and even failed at some serious points while the proposed controller kept high performance for all operating conditions. 


\section{CONCLUSION}

The feedback error learning (FEL) scheme is used to improve the small-signal stabilities of an electric power system composed of a synchronous generator connected to an infinite bus. The FEL is implemented by combining two controllers which are feed forward neural network controller and classical feedback controller with fixed gain. The electrical power system is tested in three cases of different load conditions. In general, by comparing the system dynamic responses for both CPSS and FEL, it can be clearly seen that the proposed FEL scheme has much better responses than CPSS from the view point of peak overshoot and undershoot of the generator rotor speed deviation. Furthermore, the simulation results of Case III clearly demonstrate the superiority of the proposed FEL control scheme against system uncertainties where the proposed scheme has maintained system stability at heavy leading power factor load whilst the system became unstable with conventional stabilizer although it was optimally tuned.

\section{REFERENCES}

[1] Kundur, P., Balu, N. J., \& Lauby, M. G. (1994). Power system stability and control, 7. New York: McGraw-hill.

[2] Kundur, P., Paserba, J., Ajjarapu, V., Anderson, G., Bose, A., Canizares, C., Hill, D, Tankovic, A., Taylor, C. Cutsem, T. V., \& Vittal, V. (2004). Definition and Classification of Power System Stability. IEEE Trans. Power Syst, 19, 13871401.

[3] Rivera, I. L. O., Vittal, V., Heydt, G. T., Fuerte-Esquivel, C. R., \& Angeles-Camacho, C. (2018). A dynamic state estimator based control for power system damping. IEEE Transactions on Power Systems, 33(6), 6839-6848.

[4] Zhang, C., Ke, D., Sun, Y., Chung, C. Y., \& Xu, J. (2018) Investigations of large-scale voltage-dependent loads for damping inter-area oscillations: Mechanism and robust decentralized control. IEEE Transactions on Power Systems, 33(6), 6037-6048.

[5] Steinmetz, C. P. (1920). Power control and stability of electric generating stations. Transactions of the American Institute of Electrical Engineers, 39(2), 1215-1287.

[6] Doherty, R. E. (1928). Excitation Systems Their Influence on Short Circuits and Maximum Power. Transactions of the American Institute of Electrical Engineers, 47(3), 944-956.

[7] Thompson, L. W. \& Walton, P. J. (1930). Automatic regulation of synchronous condensers equipped with super speed excitation. Transactions of the American Institute of Electrical Engineers, 49(1), 315-318.

[8] Park, R. H. (1966). U.S. Patent No. 3,234,397. Washington, DC: U.S. Patent and Trademark Office.

[9] Kimbark, E. W. (1995). Power system stability, 1. John Wiley \& Sons.

[10] Venikov, V. A. (2014). Transient Phenomena in Electrical Power Systems. International Series of Monographs on Electronics and Instrumentation, 24. Elsevier.

[11] Demello, F. P. \& Concordia, C. (1969). Concepts of synchronous machine stability as affected by excitation control. IEEE Transactions on power apparatus and systems, 88(4), 316-329.

[12] Deckmann, S. M. \& Da Costa, V. F. (1994). A power sensitivity model for electromechanical oscillation studies. IEEE Transactions on Power Systems, 9(2), 965-971.

[13] Trudnowski, D. J., Pierre, J. W., Zhou, N., Hauer, J. F., \& Parashar, M. (2008). Performance of three mode-meter block-processing algorithms for automated dynamic stability assessment. IEEE Transactions on Power Systems, 23(2), 680-690.

[14] Wies, R. W., Pierre, J. W., \& Trudnowski, D. J. (2003). Use of ARMA block processing for estimating stationary lowfrequency electromechanical modes of power systems. IEEE Transactions on Power Systems, 18(1), 167-173.

[15] Zhou, N., Pierre, J. W., Trudnowski, D. J., \& Guttromson, R. T. (2007). Robust RLS methods for online estimation of power system electromechanical modes. IEEE Transactions on Power Systems, 22(3), 1240-1249.

[16] Agrawal, U., Follum, J., Pierre, J. W., \& Duan, D. (2018). Electromechanical mode estimation in the presence of periodic forced oscillations. IEEE Transactions on Power Systems, 34(2), 1579-1588.

[17] Chevalier, S., Vorobev, P., \& Turitsyn, K. (2018). A bayesian approach to forced oscillation source location given uncertain generator parameters. IEEE Transactions on Power Systems, 34(2), 1641-1649.

[18] Zhou, J., Zheng, T., \& Gan, D. (2018). Value-Set-Based Power System Robust Stability Analysis: Further Results. IEEE Transactions on Power Systems, 34(2), 1383-1392.

[19] Canales, H. T., Torres, F. C., \& Chavez, J. S. (2014). Tuning of power system stabilizer PSS using genetic algorithms. In 2014 IEEE International Autumn Meeting on Power, Electronics and Computing (ROPEC), 1-6.

[20] Ernst, D., Mevludin, G., \& Louis, W. (2004). Power systems stability control: reinforcement learning framework. IEEE transactions on power systems, 19(1), 427-435.

[21] Mondal, D., Chakrabarti, A., \& Sengupta, A. (2020). Power system small signal stability analysis and control. Academic Press.

[22] Gibbard, M. J., Vowles, D. J., \& Pourbeik, P. (2015). Smallsignal stability, control and dynamic performance of power systems. University of Adelaide press.

[23] Gupta, A., Gurrala, G., \& Sastry, P. S. (2018). An online power system stability monitoring system using convolutional neural networks. IEEE Transactions on Power Systems, 34(2), 864-872.

[24] Rajapakse, A. D., Gomez, F., Nanayakkara, K., Crossley, P. A., \& Terzija, V. V. (2009). Rotor angle instability prediction using post-disturbance voltage trajectories. IEEE Transactions on Power Systems, 25(2), 947-956.

[25] AL-Masri, A. N., Ab Kadir, M. Z. A., Hizam, H., \& Mariun, N. (2013). A novel implementation for generator rotor angle stability prediction using an adaptive artificial neural network application for dynamic security assessment. IEEE Transactions on Power Systems, 28(3), 2516-2525.

[26] Dong, W., Xin, H., Wu, D., \& Huang, L. (2018). Small signal stability analysis of multi-infeed power electronic systems based on grid strength assessment. IEEE Transactions on Power Systems, 34(2), 1393-1403.

[27] Yan, Z. \& Xu, Y. (2018). Data-driven load frequency control for stochastic power systems: A deep reinforcement learning method with continuous action search. IEEE Transactions on Power Systems, 34(2), 1653-1656.

[28] Kawato, M., Furukawa, K., \& Suzuki, R. (1987). A hierarchical neural-network model for control and learning of voluntary movement. Biological cybernetics, 57(3), 169185.

[29] Nakanishi, J. \& Schaal, S. (2004). Feedback error learning and nonlinear adaptive control. Neural Networks, 17(10), 1453-1465

[30] Wongsura, S. \& Kongprawechnon, W. (2008). DiscreteTime Feedback Error Learning and Nonlinear Adaptive Controller. ECTI Transactions on Electrical Engineering, Electronics, and Communications, 6(2), 99-106.

[31] Toolbox, N. N. (1998). For use with MatLab ${ }^{\circledR}$ User's Guide, v. 3.0. The MathWorks. Inc., January-1998.

[32] Campa, G., Fravolini, M. L., \& Napolitano, M. (2002). A library of adaptive neural networks forcontrol purposes. In 
Proceedings. IEEE International Symposium on Computer Aided Control System Design, 115-120.

[33] Sambariya, D. K. \& Prasad, R. (2014). Robust tuning of power system stabilizer for small signal stability enhancement using metaheuristic bat algorithm. International Journal of Electrical Power \& Energy Systems, 61, 229-238.

[34] Krishan, R. \&Verma, A. (2016). Robust tuning of power system stabilizers using hybrid intelligent algorithm. In 2016 IEEE Power an energy Society General Meeting (PESGM), $1-5$

\section{Contact information:}

Sedat NAZLIBILEK, Profesor

(Corresponding author)

School of Electrical and Electronics Engineering,

Baskent University, 06810, Ankara

E-mail: snazlibilek@baskent.edu.tr

Issa $\mathrm{ALI}, \mathrm{PhD}$

Department of Electric and Electronic Engineering

Higher Institute of Science and Technology, 16328, Jadu, Libya

E-mail: essand11@yahoo.com

Alyaseh ASKIR, PhD

Department of Mechatronics Engineering,

AL- Zawia University, 16418, Zawia, Libya

E-mail: alyasehaskir@gmail.com 